\title{
Proposta de um quadricóptero com grande capacidade computacional autônoma
}

\author{
VICTOR G. S. SOUSA ${ }^{1}$, OSAMU SAOTOME ${ }^{2}$.
}

\author{
1. Engenharia Mecânica e Aeronáuitica, Programa de Sistemas Aeroespaciais e Mecatrônica, Instituto \\ Tecnológico de Aeronáutica - ITA, \\ 12.228-900 Praça Marechal Eduardo Gomes, 50, Vila das Acácia,s São José dos Campos, SP - Brasil \\ E-mails: victorgomes55@gmail.com, victor-gomes@hotmail.com
}

\author{
2. Divisão de Engenharia Eletrônica, Instituto Tecnológico de Aeronáutica - ITA, \\ 12.228-900 Praça Marechal Eduardo Gomes, 50, Vila das Acácia,s São José dos Campos, SP - Brasil \\ E-mails: osaotome@ita.br
}

\begin{abstract}
This article proposes a concept of a standalone quadcopter with an intensive communication with a remote control earth station. The onboard computer of the aircraft is responsible for flight stability, power management, autonomous navigation, capture and processing of still images and videos. The exchange of information between the command and with the aircraft is performed with data from the mission and / or captured images. Thus, an onboard computer endowed with intelligence decision, wireless communication system and a smart camera are proposed.
\end{abstract}

Keywords_ Quadcopter, UAV, Onboard Computer

Resumo - Este artigo apresenta uma proposta de conceito de um quadricóptero autônomo, com uma comunicação intensa com uma estação de controle remota em terra. O computador de bordo da aeronave é responsável pela estabilidade de voo, gerenciamento de energia, navegação autônoma, captura e processamento de imagens estáticas e de vídeos. A troca de informações da estação de comando com a aeronave é efetuada o com os dados da missão e/ou imagens capturadas. Assim, um computador de bordo dotado de inteligência de decisão, comunicação wireless e um sistema de câmera inteligente são propostos.

Palavras-chave— Quadricóptero, VANT, Computador de bordo

\section{Introdução}

O veículo aéreo não-tripulado (VANT) autônomo proposto é um helicóptero de quatro motores, alocados de forma simétrica em sua estrutura, essa aeronave é conhecida também como quadricóptero. O computador de bordo é responsável por todas as suas funcionalidades disponíveis, assim como, comunicação com a base, gerenciamento dos sensores [3], controle dos motores [1] e aquisição de imagens [5].

O quadricóptero é dotado com uma antena 2,4GHz, responsável pela troca de informação entre a base e a aeronave. Esse sistema de telemetria possibilita que a unidade terrestre de monitoramento realize cálculos paralelos aos do computador de bordo, para analisa-los e prover possíveis correções.

O sistema de sensores possui uma Inertial Measurement Unit (IMU), cinco sensores de ultra-som, um GPS, um infra-vermelho, uma câmera e medidores de tensão e corrente nos pontos de interesse. A IMU é uma unidade de medida inercial, responsável pela medição de 6 eixos, sendo 3 eixos de rotação e 3 de aceleração. O conjunto de 5 sensores de ultra-som e o infra-vermelho possibilitam a obtenção da distância dos obstáculos à aeronave nas 6 possíveis direções espaciais, sendo o sensor infra-vermelho res- ponsável pela aquisição da distância entre a aeronave e o solo. O GPS informará ao sistema a posição georeferenciada da aeronave. A câmera aquisitará imagens de alta definição para o sistema, que podem fornecer diversas informações por meio de processamento digital de imagens. Os medidores de tensão e corrente fornecem um conjunto de dados que auxiliam o sistema de telemetria no controle das funcionalidades da aeronave.

A arquitetura completa de software do computador de bordo será composta por um sistema operacional embarcado em uma plataforma Linux, utilizando um microprocessador da família Cortex A8 e um microcontrolador Cortex M3, com um aplicativo embarcado em Linguagem C. Este sistema proverá a implementação de funcionalidades, a fim de integrar todo o sistema de sensoriamento da aeronave, provendo o cumprimento de uma missão atribuída.

As missões a serem realizadas necessitarão de um sistema de navegação inteligente [2][4], que traça as possíveis rotas e gerencia o deslocamento da aeronave no percurso estipulado.

Tal sistema pode ser usado em diversos ramos de atividades, como por exemplo, em segurança, na indústria, na agricultura, em fotografia aérea, em repetidor de sinal, etc.

Atualmente alguns grupos open source, relacionadas com a prototipagem de quadricópteros, apresentam em suas páginas web modelos de aeronaves 
com um processamento relativamente baixo se comparado com o proposto, algumas possuem o seu principal foco em modelos de baixo custo, a titulo de exemplo alguns desses grupos são: KKmulticopter, Multiwii, Aeroquad, Arduino Platform.

\section{FORMULAÇÃO DO PROBLEMA}

A estabilidade de voo do quadricóptero é realizada por meio de um controle simultâneo de quatro motores do mesmo e avaliação da sua atitude através da IMU. Há necessidade da utilização de equações de controle, que possibilitam além da estabilização, a movimentação em todas as direções, garantindo o controle da estabilidade da aeronave com relação a perturbações do ambiente. Os motores do quadricóptero representam o maior consumo de energia do sistema, então quanto melhor for o controle das variáveis para o cumprimento da missão maior será o tempo de voo.

A Estação de controle enviará um pacote de dados para o quadricóptero contendo os objetivos da missão e mapas. Em princípio as primeiras orientações a aeronave ainda se encontrará em solo. O computador de bordo basicamente processará paralelamente com a base os dados enviados, de forma a obter o melhor plano de vôo para a missão. Independente da missão e em qualquer situação de vôo, o computador de bordo deverá ser capaz de manter de forma autônoma a aeronave estabilizada e um total controle no gerenciamento de energia disponível, de forma a sempre possibilitar o retorno da aeronave à base, completando ou não a missão.

Traçado o plano de vôo, eventuais obstáculos ou perturbações atmosféricas não previstas, podem surgir no percurso e a aeronave deve ser capaz de evitar colisões em tempo real, se manter estável e como prioridade, o retorno seguro à base. Essas características se fazem necessárias principalmente em missões de reconhecimento outdoor ou indoor.

Uma vez aquisitada, toda imagem é armazenada na memória, possuindo a mesma resolução. Estas necessitarão de um pré processamento para corrigir os efeitos dos possíveis ruídos. Como mencionado, o maior consumo do quadricóptero ocorre nos motores, a obtenção com alta exatidão do ponto de aquisição da imagem por meio do ajuste contínuo dos motores acarretará em um consumo excessivo da bateria, e visando uma maior economia da mesma e uma melhor adaptação teórica para casos reais, uma taxa de erro otimizada será calculada pelo computador para as três dimensões da posição de aquisição da imagem. O processamento digital das imagens captadas pela aeronave deve ser executado principalmente com base nos dados da IMU e GPS, pois possivelmente algumas imagens serão aquisitadas com alguma angulação ou com diferença de altitude.

\section{ARQUITETURA DO SISTEMA}

1) Hardware: A arquitetura de hardware do computador de bordo é composta basicamente de um microprocessador conectado a uma rede dedicada com protocolo próprio para otimização da comunicação, compartilhando assim dados com uma sala de situação também conectada a essa mesma rede. do na

O sistema do computador de bordo é apresenta-

Figura 1, que contém o digrama em blocos do sistema.

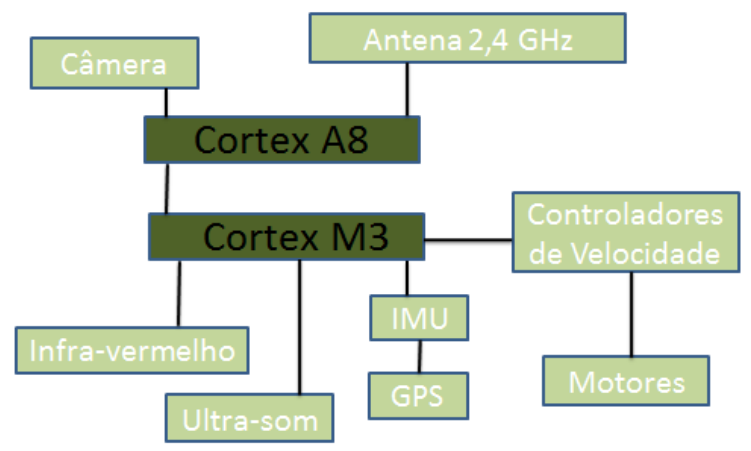

Figura 1. Arquitetura de Hardware do Computador de Bordo

O microprocessador escolhido é um Cortex A8, com uma frequência de clock igual ou superior a $600 \mathrm{MHz}$, recomendado para sistemas de alta performance e que possuem aplicações de propósito geral e de mídia, enquanto mantém um baixo consumo e um pequeno footprint. A CPU é equipada com suporte para sistemas operacionais de alto nível, acelerador gráfico, um alto desempenho na transferência de dados com controladores de memória internos e externos e periféricos conectados ao chip. Tal sistema ficará responsável pelo gerenciamento dos dados de telemetria, aquisição de imagens e transmissão dos dados pertinentes para a unidade em solo.

Para realizar o controle de gerenciamento dos sensores foi selecionado um hardware baseado em um microcontrolador NXP, que tem um núcleo ARM Cortex M3, com frequência de $96 \mathrm{MHz}$, com $512 \mathrm{~KB}$ de flash, 64 KB de RAM, bem como várias interfaces, incluindo Ethernet, dispositivo USB, CAN, SPI , $\mathrm{I}^{2} \mathrm{C}$ e outros periféricos de entrada e saída. Os barramentos de conexão do microcontrolador obedecem aos padrões de protocolos necessários para a interface com os sensores definidos.

O sistema de telemetria do computador de bordo deverá ser capaz de monitorar os dados de consumo dos nós para evitar desperdício de energia em caso de um possível surto. Todos os dados coletados durante a missão deverão ser armazenados para posterior envio a base, onde integrarão um sistema de banco de dados da aeronave.

O controle dos motores será realizado por meio de modulação por largura de pulso (PWM), onde o sistema enviará para cada driver dos motores o trem 
de pulso necessário e obterá como resposta a velocidade de rotação do rotor por meio de um sinal PWM.

Os sensores definidos para a concepção do computador de bordo estão ilustrados na Figura 2, estando descritos pelo seu tipo e protocolo de comunicação.

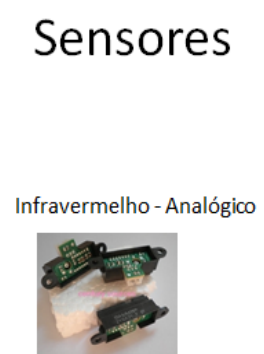

(b)

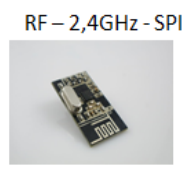

(e)

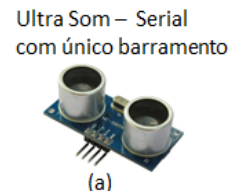

(a)

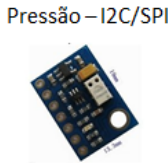

(c)

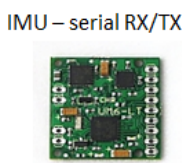

(f)
Controlador de Velocidade - PWM

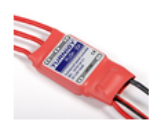

(d)

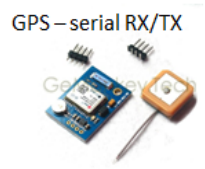

(g)
Figura 2: Sensores selecionados (a)Ultra-som HC-SR04, (b) Infravermelho GP2Y0A21, (c) Pressão MS5611, (d) controlador de Velocidade Turnigy-30A, (e) Radio Frequência NRF24101, (f) Unidade inercial UM6-LT, (g) GPS NEO-6M.

2) Software: A arquitetura de software será basicamente dois sistemas, um sistema embarcado em Linux utilizando os padrões comuns de desenvolvimento com duas camadas, aplicação e kernel-mode. Esta última é dividida em dois blocos, device drivers e tabela de dados. A camada de aplicação conterá os gerenciamentos de missão, voo, energia e imagens. A Figura 3 ilustra a arquitetura de software.

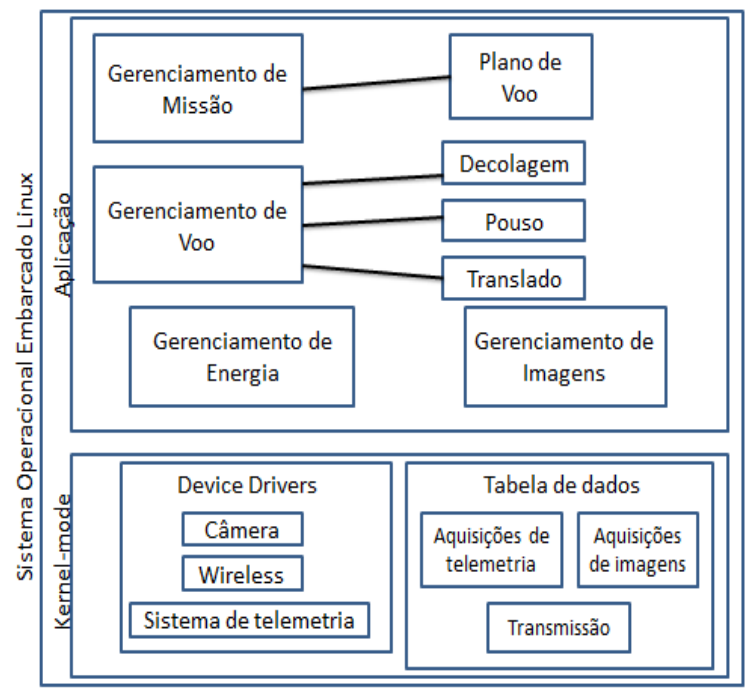

Figura 3 Arquitetura de Software do Computador de Bordo

A camada do Kernel integra a aplicação com o hardware por meio dos seus dois blocos, o device driver responsável pela integração das variáveis de interesse da camada de aplicação com as funcionalidades disponíveis do sistema de sensoriamento do computador de bordo, através de funções de configuração de hardware contidas neste bloco. As tabelas armazenam os dados coletados pelas câmeras, sensores ou os transmitidos pela antena de wireless e ainda são utilizadas como base de dados para as funções da camada de aplicação.

A camada de aplicação do software inicia seu processo após o recebimento do pacote de dados da missão. O processo de gerenciamento da missão realiza o plano de voo, com a rota prevista, dados de altitude e velocidade. O mesmo representa para o sistema o papel do piloto, onde o plano de voo prédefine as variáveis dinâmicas da aeronave.

As missões, que necessitarem do uso da câmera, terão a sua aquisição de imagens gerenciada de forma paralela de acordo com o objetivo da missão. O computador de bordo possuirá a capacidade de um pré processamento digital de imagens por meio da aplicação de filtros durante a aquisição.

Durante a execução do plano de voo, um algoritmo de gerenciamento de energia analisa o consumo da aeronave, de forma a sempre permitir a possibilidade do retorno à base no caso de uma possível falta de energia no cumprimento da missão.

O segundo sistema, responsável pelas configurações de funcionamento do microcontrolador, desenvolvido em linguagem $C$, será uma unidade de aquisição e filtragem de dados aquisitados dos sensores descritos anteriormente e de acordo com tais valores adicionados as informações de planos de voo, processadas na unidade de maior processamento, aturá nos controladores de velocidade, de modo a atender o que foi processado pelo sistema. O sistema em sua versão inicial atenderá o diagrama básico de voo autônomo ilustrado na Figura 4.

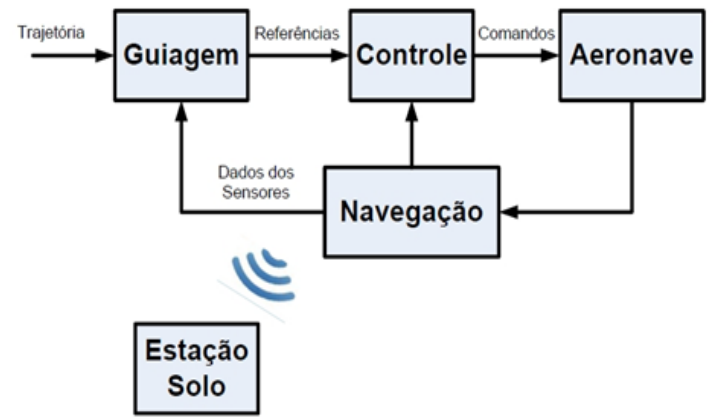

Figura 4 Sistema de controle de um piloto automático

No que se diz respeito a Estação de Solo, um estudo utilizando o Software Labview interfacenando com o hardware CortexM3 está em andamento, e proverá informações precisas a uma taxa de aquisição relativamente alta, isto é, atenderá as necessidades requeridas pelo sistema.

\section{DISCUSSÕES E TRABALHOS FUTUROS}

O sistema descrito nesse artigo é uma plataforma modular de hardware e software. Dessa forma havendo novas necessidades o sistema poderá ser implementado com as atualizações sem prejudicar as funcionalidades já desenvolvidas. 
O projeto do quadricóptero pode ser dividido em três fases: funcionalidades básicas, missões e voo em grupo. A primeira fase de testes será aplicada no sistema seguindo o conceito de hardware-in-loop, que por meio de simuladores serão testados os algoritmos, a dinâmica de voo da aeronave e as funcionalidades do próprio computador de bordo. Numa segunda etapa os testes serão aplicados ao quadricóptero preso a uma base, para possibilitar uma análise real do seu controle de estabilidade, decolagem e pouso. Tais testes laboratoriais servirão como base para a terceira etapa, onde os quadricópteros iniciarão testes com condições de voo autônomas. Ao final da primeira fase do protótipo, o sistema terá o controle das funcionalidades básicas do quadricóptero, que incluem decolagem, pouso, voos panorâmicos a baixa velocidade, aquisição de imagens e telemetria.

A segunda fase seguirá a sequência de testes da primeira, mas com foco no processamento das missões. Na primeira parte desta fase o sistema de navegação da aeronave será testado exaustivamente no simulador, os dados aqui fornecidos serviram de base para futuras simulações com a aeronave em campo. Na etapa seguinte o processamento das missões se dará simulando uma possível missão real processada no computador de bordo e paralelamente na base, mas nesse ponto o quadricóptero não realizará voos, após o processamento a aeronave enviará para a base o seu plano de voo, que será comparado ao processado na base. No final desta fase o sistema de navegação do quadricóptero estará refinado e os voos serão georeferenciados. O processamento se dará de forma paralela na base e no computador de bordo e a aeronave estará apta a realizar todos os propósitos descritos neste artigo.

A terceira fase é enquadrada como um trabalho futuro, pois a replicação da aeronave e do computador de bordo possibilita a formação de um grupo de aeronaves, a fim de realizar missões em equipe ou diversas missões independentes, que juntas alcançariam um objetivo comum. O computador de bordo realiza a sua transmissão e recepção via wireless, uma das aeronaves poderia criar uma rede para as outras se conectarem. Dessa maneira as aeronaves poderiam de forma autônoma se comunicarem, informando sobre a sua posição ou realizando transferência de pacotes de dados.

\section{Contribuição desse artigo}

A principal contribuição desse artigo é a proposta de um sistema de processamento distribuído de uma aeronave autônoma com um processamento de alta performance, uma vez que os componentes selecionados possuem uma alta resolução e uma sensibilidade considerável.

\section{Conclusão}

O quadricóptero introduzido neste artigo abrange uma arquitetura de hardware e software, que foram definidos objetivando a sua utilização em missões de VANTs autônomos. O sistema foi modelado para fornecer as condições necessárias a todas as variáveis críticas desse sistema. As arquiteturas propostas podem ser transferidas para outros tipos de veículos autônomos, através de pequenas mudanças modulares no sistema.

Devido ao uso de motores elétricos a autonomia é prejudicada, pois o sistema fica dependente de uma bateria. As tecnologias atuais de armazenamento de energia podem prover para este tipo de aeronave um tempo de voo estimado de no máximo $30 \mathrm{~min}$.

Apesar da baixa autonomia esse modelo de aeronave possui um foco em aplicações, onde o período de monitoramento necessário para uma determinada área ou ponto específico é na escala de minutos em lugares de difícil acesso por meios convencionais. Para a utilização desse equipamento o espaço necessário para decolagem e pouso é de aproximadamente $1 \mathrm{~m}^{2}$, tornando a sua utilização simples e prática.

\section{Agradecimentos}

Gostaria de agradecer ao meu orientador, prof. Dr. Osamu Saotome por todo o apoio e também por ter me ajudado durante todo o processo.

Agradecimentos ao apoio dado pelo Instituto de Tecnológico de Aeronáutica, por prover a infraestrutura necessária para os meus estudos.

\section{Referências Bibliográficas}

[1] V. Erik and S. Kamesh (2010). "Backstepping based nested multi-loop control laws for a quadrotor”, Int. Conf. Control, Automation, Robotics and Vison, Singapore.

[2] W. Liang-Chuan and W. Chih-Yu (2010). "Adaptive trajectory tracking for UAV guidance with Bayesian filtering", IEEE Conference on Industrial Electronics and Applicationsis.

[3] A. Nemra and N. Aouf (2009). "Robust INS/GPS sensor fusion for UAV Localization using SDRE nonlinear filtering”.

[4] G. Duo-Yu, Z. Cheng-Fei, G. Jiang, L. Shu-Xiao and C. Hong-Xing (2010). "Vision-Aided UAV navigation using GIS data", IEEE.

[5] L. Yuping and M. Gérard (2007). "Mapenhanced UAV image sequence registration and synchronization of multiple image sequences", IEEE. 